\title{
Menthol smoking in relation to time to first cigarette and cotinine: Results from a community-based study
}

\author{
Joshua E. Muscat ${ }^{\mathrm{a}, *}$, Hsiao-Pin Liu ${ }^{\mathrm{a}}$, Steven D. Stellman ${ }^{\mathrm{b}}$, John P. Richie Jr. ${ }^{\mathrm{a}}$ \\ ${ }^{a}$ Department of Public Health Sciences, Penn State College of Medicine, 500 University Drive, Hershey, PA 17033, United States \\ ${ }^{\mathrm{b}}$ Department of Epidemiology, Mailman School of Public Health, Columbia University, New York, NY 10032, United States
}

\section{A R T I C L E I N F O}

\section{Article history:}

Received 31 October 2011

Available online 2 April 2012

\section{Keywords:}

Nicotine

Dependence

Menthol

Cotinine

Tobacco

\begin{abstract}
A B S T R A C T
Smokers who have their first cigarette shortly after waking, an indicator of nicotine dependence, have substantially higher cotinine levels. There is controversy regarding the role of menthol in nicotine dependence. We hypothesized that menthol smokers have a shorter time to first cigarette (TTFC), and tested whether any statistical association actually reflects increased dependence by measuring nicotine uptake (e.g. cotinine) in the same group of smokers. A cross-sectional community-based study was conducted that included 495 black and white daily cigarette smokers. Results showed a trend between menthol smoking and a shorter TTFC $(P<0.04$ in blacks $)$. Menthol was not an independent predictor of cotinine or an effect modifier with TTFC on cotinine levels in blacks and whites. These results show that while menthol in tobacco is associated with an indicator of nicotine dependence in blacks, menthol was not associated with biological uptake of nicotine in black and white smokers.
\end{abstract}

(C) 2012 Elsevier Inc. All rights reserved.

\section{Introduction}

The 2009 Family Smoking Prevention and Tobacco Control Act gave the Food and Drug Administration (FDA) the authority to regulate the advertising, marketing and tobacco content of cigarettes including the characterization of tobacco by flavor. The Tobacco Products Scientific Advisory Committee (TPSAC) made no specific policy recommendations to the FDA in 2011, and left opens the question of whether menthol in cigarettes should be banned. The panel noted that menthol facilitated nicotine dependence in some groups.

The role of menthol cigarettes in nicotine addiction has been extensively studied (Ahijevych and Garrett, 2010; Heck, 2010), yet many aspects of its effects are still not well understood and remains uncertain (Hoffman and Simmons, 2011). There has been a growing interest in studying the effects of menthol on the time to first cigarette (TTFC) since this measure is associated with many of the behavioral dimensions of nicotine dependence including smoking amount (Heatherton et al., 1989; Kozlowski et al., 1994), inability to quit (Baker et al., 2007; Kabat and Wynder, 1987; Kozlowski et al., 1994; Li et al., 2011), smoking relapse

\footnotetext{
Abbreviation: TFFC, Time to first cigarette.

* Corresponding author. Address: 500 University Blvd., Penn State College of Medicine, Hershey, PA 17033, United States. Fax: +1 7175310480.

E-mail addresses: jmuscat@psu.edu (J.E. Muscat), HLIU@hes.hmc.psu.edu (H.-P. Liu), sds91@columbia.edu (S.D. Stellman), JRICHIE@hmc.psu.edu (J.P. Richie Jr.).
}

(Toll et al., 2007), tolerance (Pillitteri et al., 1997) and nighttime smoking (Bover et al., 2008). The TTFC accounts for much of the predictive validity of the Fagerstrom test for Nicotine Dependence (Baker et al., 2007).

Initial studies of menthol and TTFC found a shorter mean TTFC in menthol vs. non-menthol smokers in 95 black and white women (Ahijevych and Parsley, 1999) and in 600 black smoking cessation trial participants (Okuyemi et al., 2003). In contrast, menthol was not associated with a TTFC $\leqslant 10 \mathrm{~min}$ in 13,268 black and white participants in the Community Intervention Trial for Smoking Cessation (Hyland et al., 2002), and with a TTFC $\leqslant 30 \mathrm{~min}$ in daily smokers aged 18-24 years participating in the Tobacco Use Supplements to the Current Population Surveys (TUS CPS) (Ahijevych and Ford, 2010). Menthol was associated with a TTFC $\leqslant 30 \mathrm{~min}$ in nondaily smokers $18-24$ years old. In a separate analysis of all adult TUS CPS participants, menthol was associated with a TTFC $\leqslant 5$ min in smokers consuming 6-10 cigarettes per day (Fagan et al., 2010). Race-specific results were not presented. In a tobacco treatment clinic that studied 1699 patients, menthol was associated with a shorter TTFC (Gandhi et al., 2009). The inconsistency in the TTFC findings might be due to differences in subject characteristics such as age, race, study population (e.g. randomized trial vs. population-based), and categorical vs. continuous measures of TTFC.

Even if menthol is associated with a shorter TTFC, it is uncertain what this statistical relationship means with respect to the effects of menthol on nicotine dependence. A shorter TTFC has been 
associated with increased nicotine uptake as determined by increased levels of cotinine (Muscat et al., 2009b; Fu et al., 2011), but the effects of menthol on the relationship between TTFC and cotinine have not been established. An assessment of this association by menthol might help to understand the potential effects of menthol on nicotine uptake (Fagan et al., 2010). Statistical associations between menthol and TTFC may not be informative by itself without contextualizing these findings with respect to physiological/biochemical measures of dependence (e.g. nicotine uptake). The association between TTFC and cotinine might be expected to differ by menthol since menthol has anesthetic and pharmacologic properties that possibly result in greater tobacco smoke exposure, although a review of this literature indicated that studies are inconclusive (Hoffman, 2011; Henningfield et al., 2003; Benowitz et al., 2004). In the current study, wefirst examined the association between menthol and TTFC in a biracial middle-class community.

\section{Methods}

\subsection{The Mount Vernon CARES study}

The Mount Vernon CARES program was a community-based health promotion and education program that was conducted in conjunction with business, community, and religious leaders in the town of Mt. Vernon, NY (Muscat ref). The original focus was on cardiovascular disease screening and prevention, but expanded to a larger effort on the health effects of tobacco, including cigarette smoke exposure. Small research laboratories were established in the town for study recruitment and sample collection. The methods for smoking assessment were previously described (Muscat et al., 2009b). Briefly, all participants were interviewed using a structured questionnaire that contained detailed items on smoking habits and sociodemographics. A smoking history was obtained including for each brand, brand name, type of cigarette, age started smoking, frequency and duration of smoking. Venipuncture blood samples were processed and blood fractions were stored in a -80 degree freezer. The study was open to residents of Mt. Vernon from 1990-2001. This is a middle-class community with middle or lower income census tracts that is approximately 50\% black and $50 \%$ white, located about 15 miles north of New York City. The study was also open to other smokers who resided elsewhere and worked in Mt. Vernon, or to residents of other nearby towns who heard of the study through word of mouth or other media. Current cigarette smokers aged 18-55 years old were included and an institutionally-approved consent form was signed by all volunteers. All subjects were required to have been daily smokers and have smoked at least five cigarettes per day for one or more years. This cut-point was established to insure exclusion of nonsmokers/passively exposed smokers since tobacco smoke biomarker levels may be similar for passive and light smokers (e.g. 1-5 cigarettes) depending on how cigarettes are smoked in active smokers, and environmental factors in passive smokers such as room ventilation, the proximity to smokers and other factors (Benowitz, 1996).

Nicotine uptake was determined by measuring plasma cotinine $(\mathrm{ng} / \mathrm{mL})$. In a preliminary analysis based on data available for 252 black and white smokers, there was a significant linear trend between a shorter time to first cigarette after waking and cotinine levels. The current study updated these findings with the completion of data entry for all subjects (an additional 243 subjects for a total of 495 subjects) and an assessment of the effects of menthol.

\subsection{Statistical analysis}

All analyses were performed using Statistical Analysis System software (version 9.1.2). Descriptive statistics included means and standard deviations, proportions and percents. The time to first cigarette was ascertained using four categories: $\leqslant 15 \mathrm{~min}$, 16-30 $\mathrm{min}, 31-60 \mathrm{~min}$, and >60 min. These categories were selected to be compatible with another study using these cutpoints, and differ slightly from the Fagerstrom Test for Nicotine Dependence that has as its first two categories "within 5 min" and "630 min." The Cochran-Armitage trend test was used to determine a trend in the proportions of TTFC categories between menthol and nonmenthol smokers. Separate trend tests were also conducted by categories of race and cigarettes per day (e.g. 5-10 CPD, 11-20 CPD, and >20 CPD). For the CPD >20 category, Fisher's Exact test was calculated to compare proportions.

Linear regression analysis was performed to obtain mean cotinine levels by categories of TTFC, adjusted for the covariates cigarettes per day, sex and race. A test for linear trend in cotinine levels by TTFC was conducted using a single ordinal term for the TTFC.

The hypothesis that menthol is an effect modifier for the relationship between TTFC and cotinine was tested using log-transformed values of plasma cotinine using general linear models. We used the PROC GLM procedure to model log-transformed cotinine values. The model covariates included cigarettes per day (continuous), a squared term for cigarettes per day, menthol, age, sex, and race. A term was also included for body mass index, which was previously shown to be inversely correlated with cotinine in this data. A few subjects had missing BMI, which was imputed using the average value for men or women depending on the gender of the subject with missing data. The TTFC was modeled as an ordinal variable with four levels. To test for effect modification, we used the interaction term TTFC $*$ menthol. Models are presented that include the interaction term and one model without the interaction term. Finally, separate general linear models were run for blacks only and for whites only. Statistical tests were two-sided and significance levels were set at 0.05 , except for the CochranArmitage trend test which was one-sided and set at 0.05 significance. There was no missing data in the current analysis.

\subsection{Theory}

This setting is unique from previous studies in that it includes blacks and whites, and also allows us to study this association where environmental and sociocultural influences on smoking are similar between smokers. In addition, several previous analyses of TTFC were conducted within a smoking cessation setting, where motivational factors may have affected the findings. Secondly, if an association between menthol and TTFC exists, it needs to be determined whether menthol would affect the relationship between TTFC and cotinine. This information would then provide a stronger biological basis for assessing the effects of menthol on nicotine addiction, and could therefore be informative for policy decisions.

\section{Results}

The study included 242 men and 253 women. The mean age was $34.9 \pm 10$. More than half of all subjects $(n=259)$ smoked their first cigarette within 15 min after waking. The number of menthol and non-menthol smokers was about the same ( $n=221$ vs. 274 ).

Table 1 shows the relationship between TTFC categories by menthol status. Overall there was a trend between menthol smoking and a shorter TTFC $(P<0.02)$. When analyzed by race, the trend was significant in black subjects $(P<0.04)$. In black subjects, $61 \%$ of menthol smokers smoked within the first 15 min, compared to $44 \%$ of nonmenthol smokers. Eleven percent of menthol smokers waited more than one hour after waking, compared to $18 \%$ of nonmenthol smokers. In whites, the corresponding percentages were $52 \%$ vs. $48 \%$ (within $15 \mathrm{~min}$ ) and $11 \%$ vs. $15 \%$ (more than one hour). 
Table 1

Association between race, menthol and categories of time to first cigarette.

\begin{tabular}{|c|c|c|c|c|c|}
\hline $\begin{array}{l}\text { TTFC }^{\mathrm{a}} \\
\text { Mean TTFC }\end{array}$ & $\begin{array}{l}\leqslant 15 \mathrm{~min} \\
(\sim 7.5 \mathrm{~min})\end{array}$ & $\begin{array}{l}16-30 \mathrm{~min} \\
(\sim 23 \mathrm{~min})\end{array}$ & $\begin{array}{l}31-60 \mathrm{~min} \\
(\sim 45 \mathrm{~min})\end{array}$ & $\begin{array}{l}>60 \text { min } \\
\left(\sim 120^{b}\right)\end{array}$ & $P$-value \\
\hline \multicolumn{6}{|l|}{ All Races } \\
\hline White & $132(51.0 \%)$ & $60(63.2)$ & 41 (59.4\%) & $38(52.8 \%)$ & 0.18 \\
\hline White & 127 (49.0\%) & $35(36.8)$ & $28(40.6 \%)$ & $34(47.2 \%)$ & 0.41 (trend test) \\
\hline \multicolumn{6}{|l|}{ Whites } \\
\hline White & $28(21.2 \%)$ & $11(18.3 \%)$ & $9(22.0 \%)$ & $6(15.8 \%)$ & 0.86 \\
\hline White & $104(88.8 \%)$ & 49 (22.6\%) & $32(88.0 \%)$ & $32(84.2 \%)$ & 0.58 (trend test) \\
\hline \multicolumn{6}{|l|}{ Blacks } \\
\hline White & $102(80.3 \%)$ & 25 (71.4\%) & $22(78.6 \%)$ & $18(52.9 \%)$ & 0.01 \\
\hline White & 25 (19.7\%) & $10(17.9 \%)$ & $6(21.4 \%)$ & $16(47.1 \%)$ & $<0.01$ (trend test) \\
\hline
\end{tabular}

a TTFC: Time to first cigarette.

b Arbitrary value.

The trend was not significant in whites $(P=0.32)$. The relationship between menthol and TTFC was also stratified by sex and categories of cigarettes per day (e.g. 5-10,11-20,>20). The trend between menthol and shorter TTFC was found in both men $(P=0.01)$ and women $(P=0.14$, data not shown). In two of the three CPD categories, menthol was associated with a shorter TTFC. For the categories 5-10 and 11-20 cigarettes per day, the trend test was statistically significant $(P<0.01)$. There were only five heavy smokers (e.g. $\geqslant 21 \mathrm{CPD}$ ) that had a TTFC 30 or more minutes. In lieu of a trend test, the Fisher's exact test in this group was not significant $(P=1.0)$.

Table 2 shows the mean levels of plasma cotinine by categories of TTFC for both the current sample of 495 subjects. There was a significant linear trend in cotinine levels with a shorter TTFC. The findings are similar to that previously reported for the first 252 subjects.

Table 3 shows the results from the multivariate linear analysis of $\log$ cotinine levels. The time to first cigarette $(P<0.01)$ but not menthol $(P=0.66)$ was significantly associated with cotinine levels. The covariates age, race, BMI, and cigarettes per day were also significant predictors of cotinine. Sex was not a significant

Table 2

Mean plasma cotinine values by time to first cigarette after waking in a communitybased study.

\begin{tabular}{llll}
\hline TTFC & $\mathrm{N}$ & Plasma cotinine $(\mathrm{ng} / \mathrm{ml})$ & $95 \%$ C.I. \\
\hline & & & \\
$<15 \mathrm{~min}$ & 259 & 420 & $(390-450)$ \\
$16-30 \mathrm{~min}$ & 95 & 329 & $(282-377)$ \\
$31-60 \mathrm{~min}$ & 69 & 262 & $(205-320)$ \\
$>60 \mathrm{~min}$ & 72 & 244 & $(187-302)$ \\
\hline
\end{tabular}

Mean values are adjusted for cigarettes per day, sex and race. $P$-value for trend test in cotinine levels $P<0.01$.

Table 3

Linear regression analysis of log-scale plasma cotinine levels in a community based study.

\begin{tabular}{lcccr}
\hline Variables & Model $1 \beta$ & $P$-value & Model $2 \beta$ & $P$-value \\
\hline CPD $^{*}$ & 0.063 & $<.01$ & 0.062 & $<.01$ \\
CPD $^{2}$ & -0.0008 & $<0.01$ & -0.0008 & $<0.01$ \\
Age & 0.009 & $<0.05$ & 0.009 & $<0.05$ \\
Sex & -0.018 & 0.81 & -0.016 & 0.84 \\
Race & 0.336 & $<0.01$ & 0.331 & $<0.01$ \\
BMI & -0.025 & 0.01 & -0.026 & $<0.01$ \\
Menthol & -0.043 & 0.79 & 0.090 & 0.33 \\
TTFC & -0.236 & $<.01$ & -0.207 & $<.01$ \\
Menthol $*$ TTFC & 0.068 & 0.33 & & \\
\hline
\end{tabular}

CPD, Cigarette Per Day.

Menthol $*$ TTFC, The interaction effect of menthol cigarette and time to first cigarette. predictor. The interaction term for TTFC $*$ menthol was not significant $(P=0.33)$. Since there were only five heavy smokers $(\geqslant 21$ CPD) with a TTFC $\geqslant 31 \mathrm{~min}$, another model was run excluding heavy smokers. The TTFC $*$ menthol interaction term was also not significant in this model $(P=0.24)$.

We further conducted race-specific models of cotinine since the association between menthol and an earlier TTFC was significant only in blacks. The results were very similar to the overall model that included all subjects. In blacks and in whites, the TTFC $*$ menthol interaction term was not significant (data not shown).

The time to first cigarette is a smoking behavior that possibly reflects craving and need from overnight abstinence, but is likely less subjective and subject to recall bias than the reported urge to smoke. However compensatory smoking from just the first cigarette wouldn't account for the considerably higher levels of cotinine in early morning smokers. The TTFC appears to be an indicator of increased smoke exposure throughout the day.

\section{Discussion}

There remains considerable uncertainty concerning the effects of menthol on tobacco dependence. The time to first cigarette is an attractive endpoint for studying the effects of addiction since it is easily characterized and is associated with several nicotine dependence behaviors. Findings on menthol and TTFC have been mixed. One advantage of our study in examining the association between menthol and TTFC is that it was conducted within a community-based setting where sociocultural factors were similar among its residents. Smoking behaviors are strongly related to sociodemographics factors of individual smokers and their households such as education, income, race, sex, marital status, and age. Neighborhood characteristics such as poverty, access to cigarettes, exposure to cigarette advertisements as well as tobacco control programs also affect smoking behavior (Migliorini and Siahpush, 2006; Reid et al., 2010; Siahpush et al., 2010, 2007, 2009; Wakefield et al., 2008; Barbeau et al., 2004; Diez Roux et al., 2003; Duncan et al., 1999; Kandula et al., 2009; Reijneveld, 1998, 2002; Tseng et al., 2001). Another advantage of studying the effects of menthol at the community level is that other previous studies of menthol and TTFC were based on large geographically diverse surveys, or were conducted in smoking cessation trial participants who may have different degrees of nicotine dependence and diverse socioeconomic backgrounds. The community-based 'approach collected information directly from all subjects, whereas about $20 \%$ of TUS participants were proxies. The Mt. Vernon study also included similar numbers of black and white smokers, and while the study size was modest compared to national surveys, it allowed us to examine the effects of menthol independently of race. The characteristics of the smokers in Mt. Vernon were similar to that nationwide, in terms of the percent of whites and blacks 
who smoked menthol, and the lower frequency of daily smoking among black than white subjects. There have been mixed findings on the relationship between menthol and TTFC. Our data indicate that menthol was associated with a shorter TTFC in black smokers only.

If menthol is associated with a shorter time to first cigarette, as found at least in black participants in our study population, the significance of this associationwith respect to menthol and nicotine dependence is not clear. In clinical trials, a shorter TTFC is associated with inability to quit (Kozlowski et al., 1994; Baker et al., 2007; Toll et al., 2007) and lower tolerance (Pillitteri et al., 1997). However, there were few blacks in these TTFC studies, and presumably few menthol smokers. Consequently, it is difficult to determine whether the menthol-TTFC association actually measures an increased physiological dependence to nicotine. Therefore we further examined this relationship in relation to the biological uptake of nicotine. While some previous studies have shown that menthol smokers have higher cotinine levels (Clark et al., 1996; Caskey et al., 1993; Ahijevych and Garrett, 2004) most data including our previous report from this study found no differences (Wagenknecht et al., 1990; Ahijevych et al., 1996; Muscat et al., 2009a; Heck, 2009; Signorello et al., 2009; Caraballo et al., 2011).None of these studies considered how menthol may vary by TTFC, which itself is highly associated with increased cotinine (Muscat et al., 2009b; Etter et al., 1999).

A limitation of the study is that we included only subjects who smoked five or more cigarettes per day, a level that might be considered to include moderate or heavily dependent smokers. The findings might not be applicable to smokers who smoke few cigarettes per day and may have a lower level of nicotine dependence. Nicotine uptake was measured by cotinine which is the standard biomarker for nicotine exposure. When comparing subjects of different races or ethnicities, cotinine by itself might not be the most optimal measure of exposure. The nicotine metabolism pathway includes over a dozen metabolites (Benowitz et al., 1994). Nicotine is primarily metabolized to cotinine, which is further metabolized to trans-3'-hydroxycotinine (3HC). The ratio of $3 \mathrm{HC}$ to cotinine is about $15 \%$ higher in whites than in blacks (Kandel et al., 2007). Total nicotine metabolites might provide a better measure of nicotine uptake than cotinine only. Recall bias is always a concern in selfreported measures, although this was minimized for smoking information by having the subject show their current pack of cigarettes to the interviewer. Self-reported information on smoking frequency was very accurate we believe, where reported cigarettes per day ascertained by the initial screening process was almost identical to that provided in the subject interview. Further, subjects were asked to collect their cigarette butts for the week before the subject interview. This was used to validate self-reported smoking frequency. The correlation was very high (Muscat et al., 2009b). The TTFC is characterized by four intervals in the FTND ( 5 min or less, 6-30, 31-60, greater than 60) (Heatherton et al., 1989). The TTFC categories in our study (e.g. $1-15$ vs. $1-5 \mathrm{~min}$; $16-30$ vs. 5$30 \mathrm{~min}$ ) were based on another older study that predated the FTND categories. It is possible that the trend between menthol and TTFC might have been greater using the FTND intervals. Some studies of menthol reported TTFC as a continuous measure, and it is unclear whether this improves or decreases the validity of this self-reported measure. Another limitation of the study is the relatively small sample size for assessing the menthol-TTFC association compared to previous surveys. However, the study design was intended to measure exposure biomarkers, and included a large number of subjects with cotinine measurements.

In this study, menthol was classified according to cigarette labels. This provides a convenient dichotomous statistical measure of menthol in health outcome studies, although menthol may be present in low amounts in nonmentholated brands. The content of menthol varies from brand to brand (Celebucki et al., 2005) and some data indicate that the menthol content in a single brand has changed over time (Kreslake et al., 2008), although this has been debated. Menthol manufacturing processes vary where menthol can be sprayed onto tobacco directly or applied to the foil, cigarette packaging or filters (Heck, 2010). Menthol is intended to reduce the irritation from tobacco smoke, but the degree of its effectiveness may depend upon the manufacturing process such as the presence of "retained solvents" from the packaging that may act to increase tobacco smoke harshness. This in turn, could possibly confound studies of menthol and TTFC since the binary classification of menthol may not account for qualitative differences in menthol manufacturing.

Finally, there are many different measures of nicotine dependence although the Diagnostic and Statistical Manual criteria are not considered reliably accurate (DiFranza et al., 2010). Several psychometric scales are commonly employed in tobacco dependence research (Piper et al., 2006). Further studies might be useful to validate the current findings, using other measures of nicotine dependence in lieu of the TTFC.

\section{Conclusions}

Our findings may be useful in federal decisions to regulate the advertising, marketing and menthol content of cigarettes. There may be reasons for removing menthol cigarettes from the market as recommended by the TPSAC including its appeal to youths and increased smoking rates among young smokers, particularly African-Americans. No specific recommendations were made for banning menthol and there is no timeframe for FDA action. The current data indicate that while there is evidence that menthol is associated with a behavioral measure of nicotine dependence in black adults who are daily smokers, but also shows that this association does not implicate menthol as a factor in nicotine uptake in active adult black and white smokers.

\section{Acknowledgments}

This study was supported by a contract from the Food and Drug Administration through RTI, inc., and research grants (PO1 CA68384 and K07 CA104231) from the National Institutes of Health, National Cancer Institute and by a contract (PA-DOH 4100038714) from the Pennsylvania Department of Health. The sponsors did not have a role in the design and analysis of the study, the writing of the report, and the submission of the paper for publication. The authors report no conflict of interest.

\section{Appendix A. Supplementary data}

Supplementary data associated with this article can be found, in the online version, at http://dx.doi.org/10.1016/j.yrtph.2012.03. 012.

\section{References}

Ahijevych, K., Ford, J., 2010. The relationships between menthol cigarette preference and state tobacco control policies on smoking behaviors of young adult smokers in the 2006-07 Tobacco Use Supplements to the Current Population Surveys (TUS CPS). Addiction 105 (Suppl 1), 46-54

Ahijevych, K., Garrett, B.E., 2004. Menthol pharmacology and its potential impact on cigarette smoking behavior. Nicotine Tob. Res. 6 (Suppl 1), S17-S28.

Ahijevych, K., Garrett, B.E., 2010. The role of menthol in cigarettes as a reinforcer of smoking behavior. Nicotine Tob. Res. 12 (Suppl 2), S110-S116.

Ahijevych, K. et al., 1996. Menthol and nonmenthol cigarettes and smoke exposure in black and white women. Pharmacol. Biochem. Behav. 53, 355-360.

Ahijevych, K., Parsley, L.A., 1999. Smoke constituent exposure and stage of change in black and white women cigarette smokers. Addict. Behav. 24, 115-120. 
Baker, T.B. et al., 2007. Time to first cigarette in the morning as an index of ability to quit smoking: implications for nicotine dependence. Nicotine Tob. Res. 9 (Suppl 4), S555-S570

Barbeau, E.M. et al., 2004. Working class matters: socioeconomic disadvantage, race/ethnicity, gender, and smoking in NHIS 2000. Am. J. Public Health 94, 269278.

Benowitz, N.L., 1996. Cotinine as a biomarker of environmental tobacco smoke exposure. Epidemiol. Rev. 18, 188-204.

Benowitz, N.L. et al., 2004. Mentholated cigarette smoking inhibits nicotine metabolism. J. Pharmacol. Exp. Ther. 310, 1208-1215.

Benowitz, N.L. et al., 1994. Nicotine metabolic profile in man: comparison of cigarette smoking and transdermal nicotine. J. Pharmacol. Exp. Ther. 268, 296303.

Bover, M.T. et al., 2008. Waking at night to smoke as a marker for tobacco dependence. patient characteristics and relationship to treatment outcome. Int. J. Clin. Pract. 62, 182-190.

Caraballo, R.S. et al., 2011. Comparison of serum cotinine concentration within and across smokers of menthol and nonmenthol cigarette brands among nonHispanic black and non-Hispanic white U.S. adult smokers, 2001-2006. Cancer Epidemiol. Biomarkers Prev. 20, 1329-1340.

Caskey, N.H. et al., 1993. Rapid smoking of menthol and nonmenthol cigarettes by black and white smokers. Pharmacol. Biochem. Behav. 46, 259-263.

Celebucki, C.C. et al., 2005. Characterization of measured menthol in 48 U.S. cigarette sub-brands. Nicotine Tob. Res. 7, 523-531.

Clark, P.I. et al., 1996. Effect of menthol cigarettes on biochemical markers of smoke exposure among black and white smokers. Chest 110, 1194-1198.

Diez Roux, A.V. et al., 2003. Area characteristics, individual-level socioeconomic indicators, and smoking in young adults: the coronary artery disease risk development in young adults study. Am. J. Epidemiol. 157, 315-326.

DiFranza, J. et al., 2010. A systematic review of the Diagnostic and Statistical Manual diagnostic criteria for nicotine dependence. Addict. Behav. 35, 373-382.

Duncan, C. et al., 1999. Smoking and deprivation: are there neighbourhood effects? Soc. Sci. Med. 48, 497-505.

Etter, J.F. et al., 1999. Validity of the Fagerstrom test for nicotine dependence and of the Heaviness of Smoking Index among relatively light smokers. Addiction 94 269-281.

Fagan, P. et al., 2010. Nicotine dependence and quitting behaviors among menthol and non-menthol smokers with similar consumptive patterns. Addiction 105 (Suppl 1), 55-74.

$\mathrm{Fu}, \mathrm{M}$. et al., 2011. Association between time to first cigarette after waking up and salivary cotinine concentration. Nicotine Tob. Res. 13, 168-172.

Gandhi, K.K. et al., 2009. Lower quit rates among African American and Latino menthol cigarette smokers at a tobacco treatment clinic. Int. J. Clin. Pract. 63 , 360-367.

Heatherton, T.F. et al., 1989. Measuring the heaviness of smoking: using selfreported time to the first cigarette of the day and number of cigarettes smoked per day. Br. J. Addict. 84, 791-799.

Heck, J.D., 2009. Smokers of menthol and nonmenthol cigarettes exhibit similar levels of biomarkers of smoke exposure. Cancer Epidemiol. Biomarkers Prev. 18, 622-629.

Heck, J.D., 2010. A review and assessment of menthol employed as a cigarette flavoring ingredient. Food Chem. Toxicol. 48 (Suppl 2), S1-S38.

Henningfield, J.E. et al., 2003. Does menthol enhance the addictiveness of cigarettes? An agenda for research. Nicotine Tob. Res. 5, 9-11.

Hoffman, A.C., 2011. The health effects of menthol cigarettes as compared to nonmenthol cigarettes. Tob. Induc Dis. 9 (Suppl 1), S7

Hoffman, A.C., Simmons, D., 2011. Menthol cigarette smoking and nicotine dependence. Tob. Induc. Dis. 9 (Suppl 1), S5.

Hyland, A. et al., 2002. Mentholated cigarettes and smoking cessation: findings from COMMIT, Community Intervention Trial for Smoking Cessation. Tob. Control. $11,135-139$.
Kabat, G.C., Wynder, E.L., 1987. Determinants of quitting smoking. Am. J. Public Health 77, 1301-1305.

Kandel, D.B. et al., 2007. Urine nicotine metabolites and smoking behavior in a multiracial/multiethnic national sample of young adults. Am. J. Epidemiol. 165 901-910.

Kandula, N.R. et al., 2009. Association between neighborhood context and smoking prevalence among Asian Americans. Am. J. Public Health 99, 885-892.

Kozlowski, L.T. et al., 1994. Predicting smoking cessation with self-reported measures of nicotine dependence. FTQ, FTND, and HSI. Drug Alcohol Depend. 34, 211-216.

Kreslake, J.M. et al., 2008. Tobacco industry control of menthol in cigarettes and targeting of adolescents and young adults. Am. J. Public Health 98, 1685-1692.

Li, L. et al., 2011. Prospective predictors of quitting behaviours among adult smokers in six cities in China: findings from the International Tobacco Control (ITC) China Survey. Addiction 106, 1335-1345.

Migliorini, C., Siahpush, M., 2006. Smoking, not smoking: how important is where you live? Health Promot. J. Austr. 17, 226-232.

Muscat, J.E. et al., 2009a. Effects of menthol on tobacco smoke exposure, nicotine dependence, and NNAL glucuronidation. Cancer Epidemiol. Biomarkers Prev. 18, 35-41.

Muscat, J.E. et al., 2009b. Time to first cigarette after waking predicts cotinine levels Cancer Epidemiol. Biomarkers Prev. 18, 3415-3420.

Okuyemi, K.S. et al., 2003. Does menthol attenuate the effect of bupropion among African American smokers? Addiction 98, 1387-1393.

Pillitteri, J.L. et al., 1997. Individual differences in the subjective effects of the first cigarette of the day: a self-report method for studying tolerance. Exp. Clin. Psychopharmacol. 5, 83-90.

Piper, M.E. et al., 2006. Assessing tobacco dependence. a guide to measure evaluation and selection. Nicotine Tob. Res. 8, 339-351.

Reid, J.L. et al., 2010. Socioeconomic disparities in quit intentions, quit attempts, and smoking abstinence among smokers in four western countries: findings from the International Tobacco Control Four Country Survey. Nicotine Tob. Res. 12 (Suppl), S20-S33.

Reijneveld, S.A., 1998. The impact of individual and area characteristics on urban socioeconomic differences in health and smoking. Int. J. Epidemiol. 27 33-40.

Reijneveld, S.A., 2002. Neighbourhood socioeconomic context and self reported health and smoking: a secondary analysis of data on seven cities. J. Epidemiol Community Health 56, 935-942.

Siahpush, M. et al., 2010. Association of availability of tobacco products with socioeconomic and racial/ethnic characteristics of neighbourhoods. Public Health $124,525-529$.

Siahpush, M. et al., 2007. Association of smoking cessation with financial stress and material well-being: results from a prospective study of a population-based national survey. Am. J. Public Health 97, 2281-2287.

Siahpush, M. et al., 2009. Smokers with financial stress are more likely to want to quit but less likely to try or succeed: findings from the International Tobacco Control (ITC) Four Country Survey. Addiction 104, 1382-1390.

Signorello, L.B. et al., 2009. Racial differences in serum cotinine levels of smokers Dis. Markers 27, 187-192.

Toll, B.A. et al., 2007. Subjective reactivity to the first cigarette of the day as a predictor of smoking relapse: a preliminary study. Drug Alcohol Depend. 89 302-305.

Tseng, M. et al., 2001. Area-level characteristics and smoking in women. Am. J. Public Health 91, 1847-1850.

Wagenknecht, L.E. et al., 1990. Racial differences in serum cotinine levels among smokers in the Coronary Artery Risk Development in (Young) Adults study. Am. J. Public Health 80, 1053-1056.

Wakefield, M.A. et al., 2008. Impact of tobacco control policies and mass media campaigns on monthly adult smoking prevalence. Am. J. Public Health 98 1443-1450. 